\title{
Shear wave elastography of the upper trapezius muscle in patient with chronic neck pain: is there a correlation between elastic modulus and intensity of pain?
}

Junsheng Ge

Dapeng district of nanao people's hospital

Jun Zhang

Guangzhou University of Chinese Medicine

Zhi Jie Zhang ( $\nabla$ sportspt@163.com )

Louyang Orthopeadic Hospital of Henna

\section{Research article}

Keywords: upper trapezius, chronic neck pain, shear wave elastography, muscle elasctic properties, pain of intensity, rehabilitation

Posted Date: January 27th, 2020

DOI: https://doi.org/10.21203/rs.2.21900/v1

License: (c) (1) This work is licensed under a Creative Commons Attribution 4.0 International License.

Read Full License 


\section{Abstract}

Background Chronic neck pain is a common discomfort and the alteration of elasticity of the upper trapezius in pathological conditions is unknown. Therefore, the aims of this investigation were (1) to determine the intra- and inter-operator reliability of evaluating the elasticity of the upper trapezius by shear wave elastography in individuals with chronic neck pain; (2) to examine the alteration for the upper trapezius during cervical flexion; and (3) to explore the correlation between elasticity of the upper trapezius and pain intensity.

Methods Thirty individuals with chronic neck pain were recruited for this study. Alteration in elasticity of upper trapezius was evaluated by shear wave elastography during cervical flexion at $0^{\circ}$ and $50^{\circ}$.

Results The intra- and inter-operator reliability was excellent with the intraclass correlation coefficients ranging from 0.92 to 0.98 . An increase of $30.80 \%$ was shown in the elasticity of the upper trapezius during cervical flexion of the painful side. In addition, there was a significant positive correlation between elasticity of the upper trapezius and intensity of pain (rho=0.647, $\mathrm{P}<0.05)$.

Conclusion Shear wave elastography is a feasible tool for assessing changes of elasticity in muscles, and the findings in this investigation might be a good reference of evaluating upper trapezius elastic alterations in chronic neck pain.

\section{Background}

Neck pain is generally regarded as a common musculoskeletal discomfort and has a high incidence around the world [1]. Chronic neck pain (CNP), with a relatively long course of disease, decreases quality of life and especially leads to dysfunction $\mathbb{2} 2 \mathbb{X}$. In clinical terms, this pain is regarded as extensor muscle myalgia, and patients also have a stiffer feeling in this area. The extensor muscles in the neck region play an important role in adjusting stability of the neck and shoulder $₫ 3 \rrbracket$. Among them, the upper trapezius

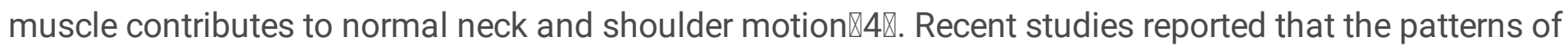
activation and fatigue of muscles were disordered when people suffered from CNP $₫ 5-6 \rrbracket$. Prolonged and unreasonable using of muscles also causes structural alteration of muscle including changed elasticity 7囚. The alterations in the biomechanical properties of muscles might explain why patients feel discomfort in the neck region. However, to date the main methods for evaluating discomfort in CNP are palpation by physicians or the subjective perception of pain by patients. There is a lack of objective technology to assess the biomechanical properties of muscles in people with CNP.

Shear wave elastography (SWE), a novel technology, is increasingly applied to assess the biomechanical properties of muscles among healthy subjects and patients with musculoskeletal disease and has been shown to be a good reliability [8-10]. Our previous studies examining the elastic properties of the patellar tendon and quadricep muscle heads were also conducted by SWE 11-13》. In addition, there are reports that the SWE can evaluate the modulation of elastic properties of muscles. In a pathological condition, increased stiffness of the upper trapezius in athletes with rotator cuff tendinopathy could be assessed 
via SWE $10 \rrbracket$. It was also detected that there were significant differences in elasticity of the biceps brachii muscles between the paretic and non-paretic side in stroke patients when using SWE 14】. Therefore, SWE is a feasible device with a high reliability to quantitatively evaluate alterations of elasticity in muscles in pathological conditions.

People in certain professions such as computer and office workers have a higher risk of suffering from CNP. They usually sit for long periods with neck flexion at a fixed angle, which easily leads to discomfort and alteration of muscle fatigue [15-16]. The upper trapezius spanning the neck and shoulder is prone to structural muscle imbalance in poor posture. It has been reported that muscle metabolism is affected by

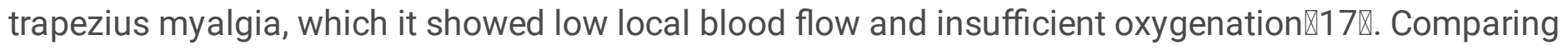
with changes of biochemical indicators, the elasticity of the upper trapezius might be impacted when people suffer from CNP. To date, no study has reported whether the elasticity of the upper trapezius in CNP is increased in poor posture. Therefore, it is necessary to assess the elastic modulus of the upper trapezius by SWE in pathological conditions. Additionally, muscle myalgia is a common symptom in neck pain. The visual analogue scale (VAS) is a relatively credible reference for assessing the intensity of chronic pain $₫ 18 \otimes$. To our knowledge, no published data shows the relationship between pain intensity and elasticity of muscle with CNP. It is therefore important to confirm what kind of correlation exists between intensity of pain and elasticity of the upper trapezius in patients with CNP.

The objectives of this investigation were (1) to determine the intra- and inter-operator reliability of evaluating the elasticity of the upper trapezius by SWE in people with CNP; (2) to examine the alteration of the upper trapezius during cervical flexion; and (3) to explore the correlation between the elasticity of the upper trapezius and pain intensity.

\section{Methods}

\section{Ethics statement}

This study was approved by the Human Subjects Ethics of the Luoyang Orthopedic Hospital of Henan Province (KY2019-001-01). The trial protocol was conducted and kept by the Declaration of Helsinki. Each subject was informed about the procedures of the experiment and provided their written informed consent prior to the experiment before study.

\section{Participant recruitment}

Thirty patients ( 9 males and 21 females) were recruited from Luoyang Orthopedic Hospital of Henan Province through a recruitment poster on April 2019 and the trail was contacted from April to July. The inclusion criteria were (1) age (39.43 \pm 14.34$)$ years with chronic nonspecific neck pain, (2) only one-sided pain experienced in the neck region for $>3$ months, (3) no therapeutic intervention before seeking medical advice, and (4) a score on the neck disability index (NDI) $\otimes 5$ [19]. The exclusion criteria were (1) other causes of neck pain such as cancer, fracture or neuropathy in the neck region and myelopathy, (2) neck 
surgery or clinical treatment for CNP, and (3) body mass index $(B M I)>30 \mathrm{~kg} / \mathrm{m}^{2}$. In addition, authors had access to information that could identify individual participants during or after data collection.

\section{Equipment of shear wave elastography}

A novel device called SWE with an Aixplorer $\rightarrow$ ultrasound unit (SuperSonic Imagine Aix-en-Provence, France) and a $50 \mathrm{~mm}$ linear ultrasound transducer (4-15 MHz) was used to evaluate the elastic modulus of the upper trapezius muscle. The principle of SWE technology is based on different shear wave velocity generated by pulses in various biological tissues [20]. Young's modulus, one of shear modulus, is generally used to indirectly reflect tissue stiffness, namely, $E=3 \rho v^{2}$, where $\rho$ represents the density of the tissue $\mathbb{2 1}$. The image of muscles is shown in B-mode and the parameter of elastic modulus is revealed in SWE-mode. Additionally, stiffer tissues were coded in red and softer areas in blue in SWE-mode区22》. The musculoskeletal mode was adopted to our one previous study for estimating the elastic properties of muscle with the temporal averaging (persistence), spatial smoothing set to medium and six, and penetration mode and the opacity at $85 \% \otimes 12 \bigotimes$. The range of the colour scale was adjusted from 0 to $200 \mathrm{kPa}$.

\section{Clinical evaluation}

The parameters of pain intensity in the VAS were calculated in this investigation. Self-perceived pain which ranged from 0 to 10 points indicated intuitively the different degrees of intensity of pain [18]. The participants were required to assess self-perceived pain via VAS. Higher VAS scores revealed the worst pain and lower scores indicated the least pain $₫ 23 \rrbracket$.

\section{Procedures}

Demographic information such as age, gender, weight, height, body mass index and weekly exercise hours were recorded before the experiment. All subjects were required to complete the assessment reports of NDI and VAS by themselves.

\section{Measurement of upper trapezius elastic modulus}

In the process of the difference analysis, the modulation of upper trapezius elastic properties was assessed by SWE during cervical flexion at $0^{\circ}$ and $50^{\circ}$ in the painful side. The room temperature was maintained at $25^{\circ} \mathrm{C}$ throughout all tests. All participants sat on a chair with shoulders in a neutral position and knees in 90-degree flexion. Before assessment, the subjects were allowed to have a $5 \mathrm{~min}$ rest in the sitting position. The angle of cervical flexion was measured by a new iPhone application named Goniometer Pro [24], which is a measuring tool of cervical spine range of motion with a good

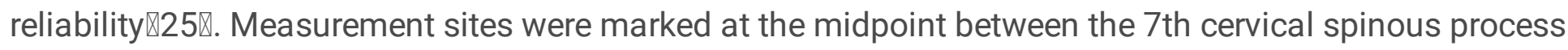
and the acromion using a marker 26 . Before scanning, ultrasound gel was applied to the skin around the probe location. In B-mode, the probe was placed perpendicularly to the skin and adjusted slightly for obtaining a clear image. Once the image without a muscle anisotropic artefact was determined, we switched to SWE-mode to quantify the elastic modulus of the upper trapezius muscle. (Fig. 1). The size of 
the circular regions of interest (ROIs) was defined as the thickness of the upper trapezius $₫ 26 \rrbracket$. Three measurements were taken and the mean was used in this study.

\section{Intra- and inter-reliability test}

The upper trapezius elastic properties in non-painful side were evaluated by SWE. The non-painful side was involved in the intra- and inter-operator reliability tests. The operators have extensive experience in assessing muscles elastic properties using SWE. For assessing the intra-operator reliability, 8 subjects were examined by operator $A$ using SWE at $0^{\circ}$ of neck flexion. The same subjects were evaluated again by operator $\mathrm{A} 5$ days later. For evaluation of inter-operator reliability, all subjects were assessed by both operators once, with a 30-minute interval. The operators were blinded to the results of measurements during the test. After completing the measurement task at each angle, participants were invited to relax for 2 minutes.

\section{Statistical analysis}

SPSS Version 19.0 software (SPSS Inc, Chicago, IL) was used for data analysis. The demographic information was calculated by descriptive statistics. A paired t-test was performed to compare mean elastic modulus of the upper trapezius between $0^{\circ}$ and $50^{\circ}$ of cervical flexion in the painful side. The intra- and inter-operator reliability was determined by calculating intraclass correlation coefficient (ICC) with $95 \% \mathrm{Cl}$. The intra-operator reliability was evaluated using the ICC $(3,1)$ (two-way mixed effect model, consistency) and the ICC $(2,2)$ (two-way random effect model, absolute agreement) was used to assess the inter-operator reliability. The standard error of measurement (SEM) was computed by the formula: SEM $=$ standard deviation $x$

, and minimal detectable change was calculated by the formula: $\mathrm{MDC}=1.96 \times \mathrm{SEM} \times$ . Bland-Altman plots further indicated the degree of agreement for evaluating reliabilities of SWE technique, which identifies the systematic error [27]. The correlation between elasticity of the upper trapezius and scores of the VAS was demonstrated by Spearman's rank correlation test. All measurement data were expressed as mean \pm standard deviation, and $\mathrm{P}<0.05$ indicated a significant level.

\section{Results}

\section{Demographic data}

Demographic information for all participants regarding age, weight, height, body mass index (BMI) and weekly exercise hours are depicted in Table 1. The clinical assessment reports regarding NDI and VAS are also presented in Table 1.

\section{Intra- and inter-operator reliability}

The related statistical parameters involving intra- and inter-operator reliability in the non-painful side during cervical flexion at $0^{\circ}$ are revealed in Table 2 . The ICC of intra-operator reliability was 
$0.92(95 \% \mathrm{Cl}: 0.67 \sim 0.98)$, and $0.98(95 \% \mathrm{Cl}: 0.91 \sim 0.99)$ for inter-operator reliability. The values of SEM ranged from 0.73 to $1.71 \mathrm{kPa}$ and the MDC ranged from 2.02 to $4.74 \mathrm{kPa}$.

In addition, Bland and Altman plot for intraoperator reliability is shown in Fig. 2a with the mean difference was $0.24 \mathrm{kPa}$ and $95 \%$ limits of agreement were -4.36 to $4.83 \mathrm{kPa}$. The plot for interoperator reliability is indicated in Fig. $2 \mathrm{~b}$. The mean difference $0.59 \mathrm{kPa}$ and $95 \%$ limits of agreement were -1.37 to $2.55 \mathrm{kPa}$. No systematic bias between two measurements was revealed in Bland and Altman plots.

Elasticity of upper trapezius at different degrees of cervical flexion in painful side

Figure 3 depicts significant differences of elastic modulus of the upper trapezius between $0^{\circ}$ and $50^{\circ}$ of cervical flexion in the painful side (Pख0.05). The mean value of the upper trapezius stiffness was $62.84 \pm$ $13.80 \mathrm{kPa}$ at $0^{\circ}$ of cervical flexion. By comparison, the stiffness was $90.75 \pm 19.94 \mathrm{kPa}$ at $50^{\circ}$ of cervical flexion, with an increase of $30.80 \%$.

\section{Correlation between elastic modulus of upper trapezius and scores of the VAS}

In subjects with CNP, Spearman's rank correlation tests were used to indicate the relationship between elasticity of the upper trapezius and intensity of pain, and there was a significant positive correlation (rho $=0.647, P \llbracket 0.05$ ) shown in Fig. 4 . It is viewed that the stiffer upper trapezius, the greater the intensity of pain.

\section{Discussion}

This study demonstrated an excellent reliability of SWE to quantify the elasticity of the upper trapezius in participants with CNP. We also noted that the elastic modulus of upper trapezius altered at different angles of cervical flexion in this investigation. Additionally, there was a significant positive correlation between the elastic property of upper trapezius and the intensity of pain among the subjects with CNP.

\section{Reliability}

In reliability tests, the parameters about the values of ICC, SEM and MDC suggest respectively the level of consistency, which demonstrates the SWE is an applicable tool with high precision. The excellent reliabilities were shown in assessing the elasticity of muscles in healthy and pathological conditions using SWE. For instance, the values of ICC ranged from 0.89 to 0.98 when quantifying properties of gastrocnemius at different postures with SWE for healthy participants [28]. For shoulder muscles such as the deltoid, supraspinatus and infraspinatus, the reliabilities of SWE were also excellent when the ICCs are larger than $0.85 \otimes 29 \bigotimes$. Furthermore, several other published studies about reliability involved assessing muscles in pathological conditions by SWE, and the reliability levels were also satisfactory. It has been reported that the biceps brachii muscles in patients with Parkinson disease were examined by SWE, and the ICCs were 0.74 and 0.78 for inter- and intra-observer variation $\varangle 30 \bigotimes$. The higher ICC values could reflect 
the higher level of consistency. In this investigation, the upper trapezius in people with CNP was quantified by SWE, and the ICCs (intra- and inter reliability: 0.92 and 0.98 ) are acceptable and relatively consistent with the previous studies. In addition, the values of ICC for inter-operator reliability were higher than the intra-operator reliability in this investigation. Analysing the possible explanation that made the differences, five days from the first measurement of the interval for the same operator might be a dominant factor, the amount of exercise, and other external factors in this five-day period may have influenced the accuracy of the experiment. Indexes of SEM and MDC represent respectively the degrees of dispersion of measurements and the true change. The smaller values of SEM and MDC indicate the excellent reliability. In our study, the SEM was more than $0.73 \mathrm{kPa}$ and the MDC was more than $2.02 \mathrm{kPa}$. Leong et al. suggest the smallest SEM and MDC were $1.45 \mathrm{kPa}$ and $2.71 \mathrm{kPa}$ when evaluating the upper trapezius among healthy people by SWE囚31囚. With a different device called MyotonPro to quantify lumbar paraspinal muscles in individuals with chronic low back pain, the SEM and MDC (SEM > 7.91N/m, and $M D C>21.93 \mathrm{~N} / \mathrm{m}$ ) also revealed an acceptable reliability $\llbracket 32 \bigotimes$. Comparing with these studies, the reasons for the differences can be analysed that the evaluation tools, muscles and the study population were inconsistent. The values of SEM and MDC are credible evidence to testify that the SWE is a feasible tool with high reliability in assessing the properties of muscles.

In addition, the Bland-Altman plots were exhibited in our study for further verifying consistency. As seen in Fig. 2, the $95 \%$ confidence limits, means and the distribution of points in plots were shown intuitively in picture, and all the points were within the $95 \%$ confidence limits. Therefore, the consistency in this investigation is satisfactory.

\section{Alteration at different degrees of cervical flexion}

There was an augment of $30.80 \%$ in elasticity of the upper trapezius in the painful side with neck flexion in this investigation, of which the average values were $62.84 \pm 13.80 \mathrm{kPa}$ at $0^{\circ}$ of cervical flexion and $90.75 \pm 19.94 \mathrm{kPa}$ at $50^{\circ}$. This is the first study to estimate alterations of elasticity of muscle in patients with CNP. There are insufficient data in published studies to make a comparison. Comparing with data in healthy individuals $(17.11 \pm 65.82 \mathrm{kPa})$, the elasticity of the upper trapezius in this study was stiffer, which was a significant increase of $72.77 \%$ [31]. Moreover, one study assessing the upper trapezius with myofascial trigger points indicated that the mean of shear modulus was $13.56 \mathrm{kPa}$ at rest position and a

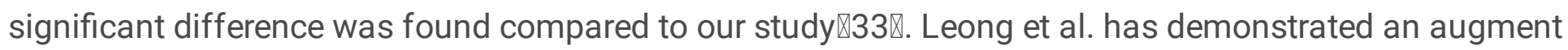
$20 \%$ of the upper trapezius by SWE among subjects with rotator cuff tendinopathy than the healthy control groups $₫ 10 \bigotimes$. In addition, increased elastic modulus of the upper trapezius in pathological conditions is also explained at the molecular level. Cornachione et al showed that the increase of $\mathrm{Ca}^{2+}$ concentration can lead to the changes of troponin configuration, the myofilament traction, and the augment of myosin globulin complex protein, which further affected the stiffness of the sarcomere $₫ 34 \rrbracket$. Based on these findings, it can be shown that the elastic modulus of the upper trapezius is increased in patients with CNP and the alterations can be quantified by SWE.

The upper trapezius maintains the stability of the cervical spine. The overload of muscles in poor posture can easily lead to an imbalance of neck extensor muscles and the poor posture might raise the risk of 
CNP. A $30.80 \%$ increase in elastic modulus of the upper trapezius with neck flexion is obvious evidence in this investigation to confirm this explanation. Lee et al. studied the relationship between activity of the upper trapezius and posture when a using smart phone. They found the alterations of muscle by electromyography (EMG) during cervical flexion: The fatigue of the upper trapezius was $-0.2 \pm 1.3 \mathrm{~Hz}$ at $0^{\circ}$ neck flexion and increased to $-3.5 \pm 5.6 \mathrm{~Hz}$ at $50^{\circ}$ [16]. Furthermore, for people with overhead work, the pressure-pain threshold of the upper trapezius was decreased by $1.4 \mathrm{lb}$ after overhead work, which it verified that the ability of tolerance of pain was reduced when people had neck pain $₫ 35 \rrbracket$. The changes of these parameters indicate that the degrees of cervical flexion could influence the biomechanical characteristics of the upper trapezius. And this finding regarding the changes of elasticity of muscle could provide reliable evidence to suggest people not keep poor posture for long periods.

\section{Correlation between elastic modulus of upper trapezius and pain intensity}

One of the main findings from this investigation was a significant positive correlation between elastic modulus of the upper trapezius and intensity of pain, which the stiffer muscles, the greater intensity of pain. Patients with CNP usually have symptoms of muscle tension accompanied by pain. One previous study has detected that this hyperalgesia was associated with glutamate and prostaglandin E2, which the release of these inflammatory substances and neurotransmitters stimulated the pain receptors to pain [36]. Sjøgaard et al. proposed that the muscle metabolism altered in trapezius myalgia. Lower local blood flow was revealed, and oxygenation of muscle was also slightly insufficient $₫ 17 \rrbracket$. They suggested that modulation of muscle metabolism may account for the higher lactate, pyruvate and pain responses. Therefore, the causes of intensity of pain might be the biochemical changes of muscles in the neck region. In addition, the intensity of pain could be evaluated by the VAS, which revealed a high reliability in chronic pain $\triangle 18 \otimes$. And assessing the intensity of pain in patients with CNP is an essential reference to provide a professional rehabilitation program.

In participants with CNP, the painful region had a stiffer muscle and a higher score of VAS. These studies about correlation are scientific guidance for physicians to diagnosis and treatment. For example, our previous study has demonstrated that elastic properties of the patellar tendon in athletes with unilateral patellar tendinopathy, and there was a significant correlation between elastic modulus of tendon and functional disability, which it could provide data to estimate whether sportsmen continue their athletic career [11]. A descriptive cross-sectional study about chronic nonspecific neck pain observed that the pressure pain thresholds were associated with NDI in the suboccipital muscle, which indicated a negative correlation $(r=-0.288 ; P<0.05) \otimes 37 \rrbracket$. Gonçalves et al. also found a negative correlation between the pressure pain thresholds and NDI in evaluating the upper trapezius in individuals with neck pain. These findings are useful to explore the mechanism of chronic pain $₫ 38$. The correlation between intensity of pain from patients and the elastic modulus of upper trapezius in this investigation could be a feasible reference to help rehabilitation therapists diagnose CNP with a more comprehensive assessment. 
Chronic neck pain is a common disease in the general population. Findings in this study suggest that the SWE with a high reliability can be used to assess muscle in pathological conditions. And it is expected to be an effective measurement to evaluate quantitatively the musculoskeletal system in physiological and pathological conditions in the future.

\section{Limitations}

There were some limitations in this investigation. (1) Only the VAS scale was selected for the study without taking into account the subjectivity of this scale. (2) We only measured one site on the upper trapezius, however, it cannot reflect the whole condition for the upper trapezius. It is worthy to explore differences of stiffness values in different parts of the upper trapezius. (3) This study only analysed the correlation between elastic modulus of the upper trapezius and the intensity of pain, therefore, the next centre of experiment is considered to concentrate on relationship with the neck disability index.

\section{Conclusion}

The present study indicated the alterations of the upper trapezius elastic properties by SWE among patients with CNP during cervical flexion. The elasticity of the upper trapezius is associated with the intensity of pain, revealing a positive correlation in chronic neck pain. In addition, SWE, a novel technology for providing relatively standard elastic parameters of biological tissues, has a good repeatability. In addition, the information of modulation of upper trapezius elastic properties in might be a good reference for the prevention and rehabilitation of CNP.

\section{Abbreviations}

CNP

chronic neck pain; SWE:Shearwave elastography; NDI:Neck disability idex; VAS:visual analogue scale; ROI:region of interest; ICC:intraclass correlation coefficient; SEM:standard error of measurement; MDC:minimal detectable change; EMG:eletramyography.

\section{Declarations}

\section{Informed consent}

Written informed consent was obtained from all subjects invovled in the study.

\section{Authors' contributions}

ZJZ conceived and designed research; JSG and JZ collected data and conducted research; JSG, JZ and ZJZ analyzed and interpreted data; JSG and JZ wrote the initial paper; ZJZ revised the manuscript; All authors read and approved the final manuscript.

\section{Competing Interests}


The authors declare that they have no competing interests.

\section{Funding}

The study was not funded by any research funding.

\section{Ethics approval and consent to participate}

This study was approved by the Human Subjects Ethics of the Luoyang Orthopedic Hospital of Henan Province (KY2019-001-01). The trial protocol was conducted and kept by the Declaration of Helsinki. Each subject was informed about the procedures of the experiment and provided their written informed consent prior to the experiment before study.

\section{Consent for publication}

All data published here are under the consent for publication.

\section{Availability of data and materials}

The datasets generated and analyzed during the current study are available from the corresponding author on reasonable request.

\section{References}

1. Hoy DG, Protani M, De R, Buchbinder R. The epidemiology of neck pain. Best Pract Res Clin Rheumatol. 2010;24(6):783-92.

2.

Tsang SMH, Szeto GPY, Xie YF, Lee RYW. Association of electromyographic activation patterns with pain and functional disability in people with chronic neck pain. Eur J Appl Physiol. 2018;118:1-12.

3.

Dieterich AV, Andrade RJ, Sant GL, Falla D, Petzke F, Hug F. Shear wave elastography reveals different degrees of passive and active stiffness of the neck extensor muscles. Eur J Appl Physiol. 2016;117(1):18.

4.

Johnson G, Bogduk N, Nowitzke A, House D. Anatomy and actions of the trapezius muscle. Clin Biomech. 1994;9:44-50. 
5.

Falla D, Rainoldi A, Merletti R, Jull G. Myoelectric manifestations of sternocleidomastoid and anterior scalene muscle fatigue in chronic neck pain patients. Clin Neurophysiol. 2003;114(3):488-95.

6.

Falla D, Bilenkij G, Jull G. Patients with chronic neck pain demonstrate altered patterns of muscle activation during performance of a functional upper limb task. Spine. 2004;29(13):1436-40.

7.

Lieber RL, Roberts TJ, Blemker SS, Ssm L, Herzog W. Skeletal muscle mechanics, energetics and plasticity. Journal of Neuroengineering Rehabilitation. 2017;14(1):108.

8.

Taljanovic MS, Gimber LH, Becker GW, Latt LD, Klauser AS, Melville DM, Gao L, Witte RS. Shear-wave elastography: basic physics and musculoskeletal applications. Radiographics. 2017;37(3):855-70. 9.

Hatta T, Giambini H, Itoigawa Y, Hooke AW, Sperling JW, Steinmann SP, Itoi E, An KN. Quantifying extensibility of rotator cuff muscle with tendon rupture using shear wave elastography: a cadaveric study. J Biomech. 2017;61:131-6.

10.

Leong H, Hug F, Fu SN. Increased upper trapezius muscle stiffness in overhead athletes with rotator cuff tendinopathy. Plos One. 2016;11(5):e0155187.

11.

Zhang ZJ, Ng GY, Lee WC, Fu SN. Changes in morphological and elastic properties of patellar tendon in athletes with unilateral patellar tendinopathy and their relationships with pain and functional disability. Plos One. 2014;9:e108337.

12.

Zhang ZJ, Ng GY, Lee WC, Fu SN. Increase in passive muscle tension of the quadriceps muscle heads in jumping athletes with patellar tendinopathy. Scandinavian Journal of Medicine Science in Sports. 2016;27:1099-104.

13.

Zhang ZJ, Fu SN. Shear elastic modulus on patellar tendon captured from supersonic shear imaging: correlation with tangent traction modulus computed from material testing system and test-retest reliability. Plos One. 2013;8(6):e68216.

14.

Wu CH, Ho YC, Hsiao MY, Chen WS, Wang TG. Evaluation of post-stroke spastic muscle stiffness using shear wave ultrasound elastography. Ultrasound in Medicine Biology. 2017;43:1105-11.

15.

Rasim UHM, Ali SS, Rasheed A, Khan M. Frequency and associated risk factors for neck pain among software engineers in karachi, Pakistan. Journal of Pakistan Medical Students. 2017;67:1009-12. 16.

Lee S, Lee D, Park J. Effect of the cervical flexion angle during smart phone use on muscle fatigueof the cervical erector spinae and upper trapezius. Journal of Physical Therapy Science. 2015;27(6):1847-49. 
17.

Sjøgaard G, Rosendal L, Kristiansen J, Blangsted AK, Skotte J, Larsson B, Gerdle B, Saltin B, Sagaard K. Muscle oxygenation and glycolysis in females with trapezius myalgia during stress and repetitive work using microdialysis and NIRS. Eur J Appl Physiol. 2010;108(4):657-69.

18.

Carlsson AM. Assessment of chronic pain. I. aspects of the reliability and validity of the visual analogue scale. Pain. 1983;16:87-101.

19.

Sherman KJ, Cook AJ, Wellman RD, Hawkes RJ, Kahn JR, Deyo RA, Cherkin DC. Five-week outcomes from a dosing trial of therapeutic massage for chronic neck pain. Annals of Family Medicine. 2014;12(2):11220.

20.

Bercoff J, Tanter M, Fink M. Supersonic shear imaging: a new technique for soft tissue elasticity mapping. IEEE Trans Ultrason Ferroelectr Freq Control. 2004;51(4):396-409.

21.

Tanter M, Bercoff J, Athanasiou A, Deffieux T, Fink M. Quantitative assessment of breast lesion viscoelasticity: initial clinical results using supersonic shear imaging. Ultrasound in Medicine Biology. 2008;34(9):1373-86.

22.

Doherty JR, Trahey GE, Nightingale KR, Palmeri ML. Acoustic radiation force elasticity imaging in diagnostic ultrasound. IEEE Transactions on Ultrasonics Ferroelectrics Frequency Control. 2013;60(4):685-701.

23.

Shin YJ, Kim WH, Kim SG. Correlations among visual analogue scale, neck disability index, shoulder joint range of motion, and muscle strength in young women with forward head posture. Journal of Exercise Rehabilitation. 2017;13(4):413-17.

24.

Pourahmadi MR, Bagheri R, Taghipour M, Takamjani IE, Sarrafzadeh J, Mohseni-Bandpei MA. A new iPhoneÂß application for measuring active craniocervical range of motion in patients with non-specific neck pain: a reliability and validity study. The Spine Journal. 2017;18:447-57.

25 .

Zhang J, Yu JF, Liu CL, Tang CZ, Zhang ZJ. Modulation in Elastic Properties of Upper Trapezius with Varying Neck Angle. Appl Bionics Biomech. 2019.

26.

Ballyns J, Turo D, Otto P, Shah J, Hammond J, Gebreab T, Gerber HL, Sikdar S. Office-Based Elastographic Technique for Quantifying Mechanical Properties of Skeletal Muscle. J Ultrasound Med. 2012;31(8):1209-19.

27.

Bland JM, Altman DG. A note on the use of the intraclass correlation coefficient in the evaluation of agreement between two methods of measurement. Computers in Biology Medicine. 1990;20(5):337-40. 
28.

Jeon M, Youn K, Yang S. Reliability and quantification of gastrocnemius elasticity at relaxing and at submaximal contracted condition. Medical ultrasonography. 2018;20:342-47.

29.

Kim K, Hwang HJ, Kim SG, Lee JH, Jeong WK. Can shoulder muscle activity be evaluated with ultrasound shear wave elastography? Clinical Orthopaedics Related Research. 2018;476(6):1276-83.

30.

Du LJ, He W, Cheng LG, Li S, Pan YS, Gao J. Ultrasound shear wave elastography in assessment of muscle stiffness in patients with parkinson's disease: a primary observation. Clin Imaging. 2016;40(6):1075-80.

31.

Leong HT, Ng GY, Leung VY, Fu SN. Quantitative estimation of muscle shear elastic modulus of the upper trapezius with supersonic shear imaging during arm positioning. Plos One. 2013;8(6):e67199.

32.

Hu X, Lei D, Li L, Leng Y, Yu Q, Wei X, Lo WLA. Quantifying paraspinal muscle tone and stiffness in young adults with chronic low back pain: a reliability study. Sci Report. 2018;8:14343.

33.

Maher RM, Hayes DM, Shinohara M. Quantification of dry needling and posture effects on myofascial trigger points using ultrasound shear-wave elastography. Arch Phys Med Rehabil. 2013;94(11):2146-50. 34.

Cornachione AS, Rassier DE. A non-cross-bridge, static tension is present in permeabilized skeletal muscle fibers after active force inhibition or actin extraction. Am J Physiol Cell Physiol. 2012;302(3):566-74. 35 .

Shin SJ, An DH, Oh JS, Yoo WG. Changes in pressure pain in the upper trapezius muscle, cervical range of motion, and the cervical flexion-relaxation ratio after overhead work. Ind Health. 2012;50(6):509-15. 36.

Flodgren GM, Crenshaw AG, Alfredson H, Fahlström M, Hellström FB, Bronemo L, Djupsjöbacka M. Glutamate and prostaglandin E2, in the trapezius muscle of female subjects with chronic muscle pain and controls determined by microdialysis. Eur J Pain. 2005;9(5):511-15.

37 .

Lopez-De-Uralde-Villanueva I, Beltran-Alacreu H, Fernandez-Carnero J, Kindelan-Calvo P. La TR.

Widespread pressure pain hyperalgesia in chronic nonspecific neck pain with neuropathic features: a descriptive cross-sectional study. Pain Physician. 2016;19(2):77-88.

38.

Gonçalves MC, Chaves TC, Florencio LL, Carvalho GF, Dach F, Fernández-De-Las-Penãs C, BevilaquaGrossi D. Is pressure pain sensitivity over the cervical musculature associated with neck disability in individuals with migraine. J Bodyw Mov Ther. 2015;19(1):67-71.

\section{Tables}


Table 1 Demographic information of subjects (30 patients)

\begin{tabular}{lc}
\hline & Mean \pm SD \\
\hline Age(years) & $39.43 \pm 14.34$ \\
Weight $(\mathrm{kg})$ & $63.63 \pm 10.05$ \\
\hline Height(m) & $1.66 \pm 0.07$ \\
\hline Body Mass Index $\left(\mathrm{kg} / \mathrm{m}^{2}\right)$ & $23.41 \pm 2.78$ \\
\hline Weekly exercise hours & $22.94 \pm 2.59$ \\
\hline Scores of VAS & $4.97 \pm 1.77$ \\
\hline Scores of NDI & $10.07 \pm 2.98$ \\
\hline
\end{tabular}

SD: Standard deviation

Table 2 Intra-and inter reliability of SWE in evaluating upper trapezius

\begin{tabular}{|c|c|c|c|c|c|}
\hline & Mean \pm SD & SEM & MDC & & \\
\hline $\begin{array}{l}\text { Operator A } \\
\text { in test } 1\end{array}$ & $36.25 \pm 6.06$ & 1.71 & 4.74 & $\mathrm{ICC}^{\mathrm{a}}(95 \% \mathrm{CI})$ & $0.92(0.67 \sim 0.98)$ \\
\hline $\begin{array}{l}\text { Operator A } \\
\text { in test } 2\end{array}$ & $36.85 \pm 5.80$ & 1.64 & 4.55 & $\operatorname{ICC}^{\mathrm{b}}(95 \% \mathrm{CI})$ & $0.98(0.91 \sim 0.99)$ \\
\hline Operator B & $36.51 \pm 5.16$ & 0.73 & 2.02 & & \\
\hline
\end{tabular}

ICC: Intra-class correlation coefficient; CI: Confidence interval; SEM(kPa): Standard error of measurement; MDC(kPa): Minimal detectable change; SD(kPa): Standard deviation kPa: kilo Pascal; a: Intra -operator reliability; b: Inter -operator reliability

\section{Figures}

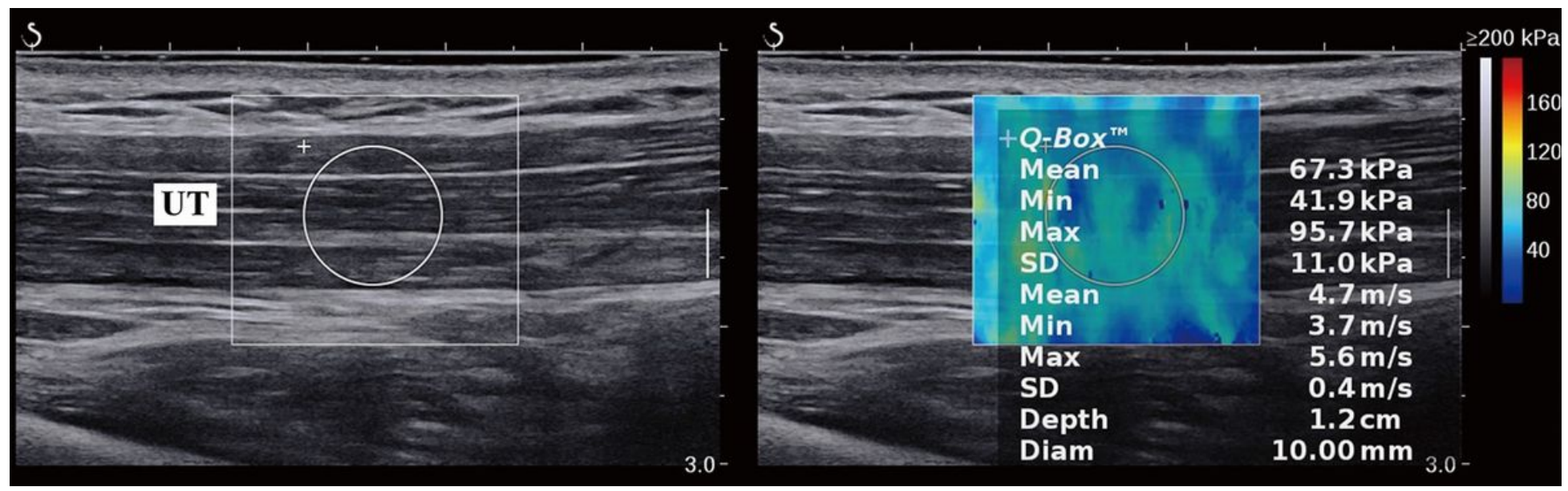


Figure 1

Shear wave elastography maps for the upper trapezius muscle. (UT: upper trapezius).
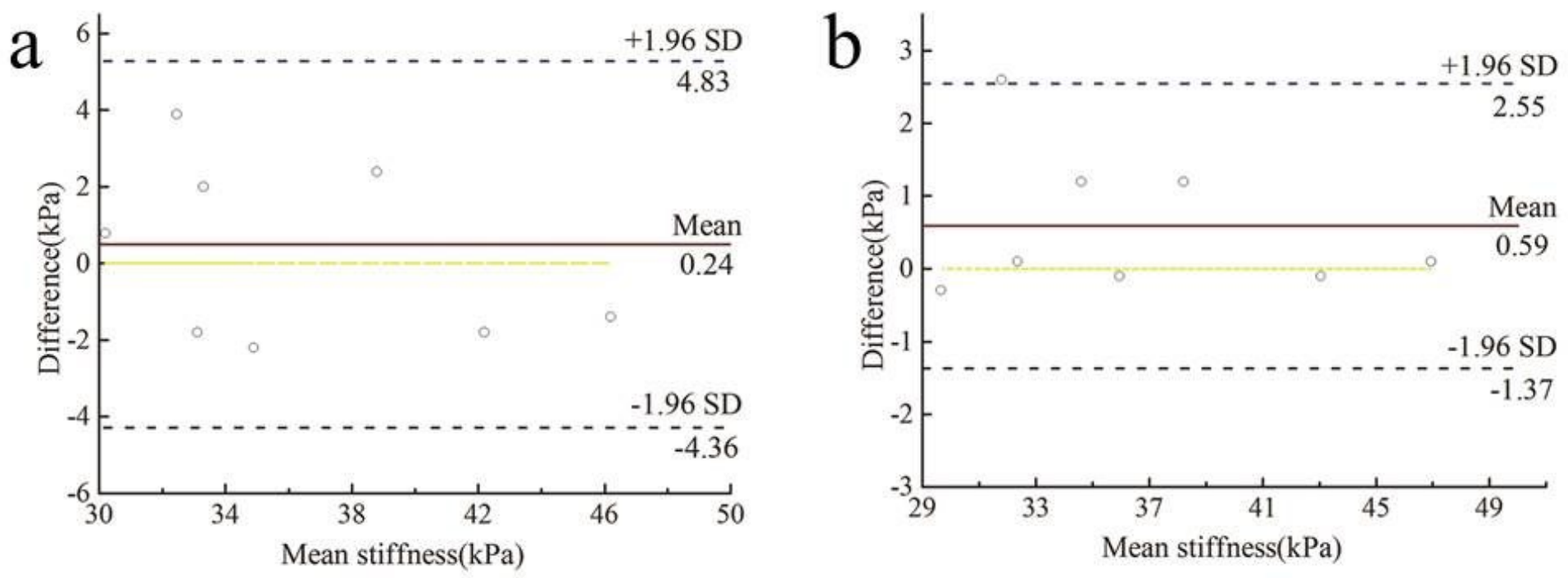

Figure 2

Bland-Altman plots of reliabilities for measuring the upper trapezius at $0^{\circ}$ cervical flexion (A and $B$ ). (A)the intraoperator reliability of assessing upper trapezius stiffness. (B)the interoperator reliability of assessing upper trapezius stiffness. 


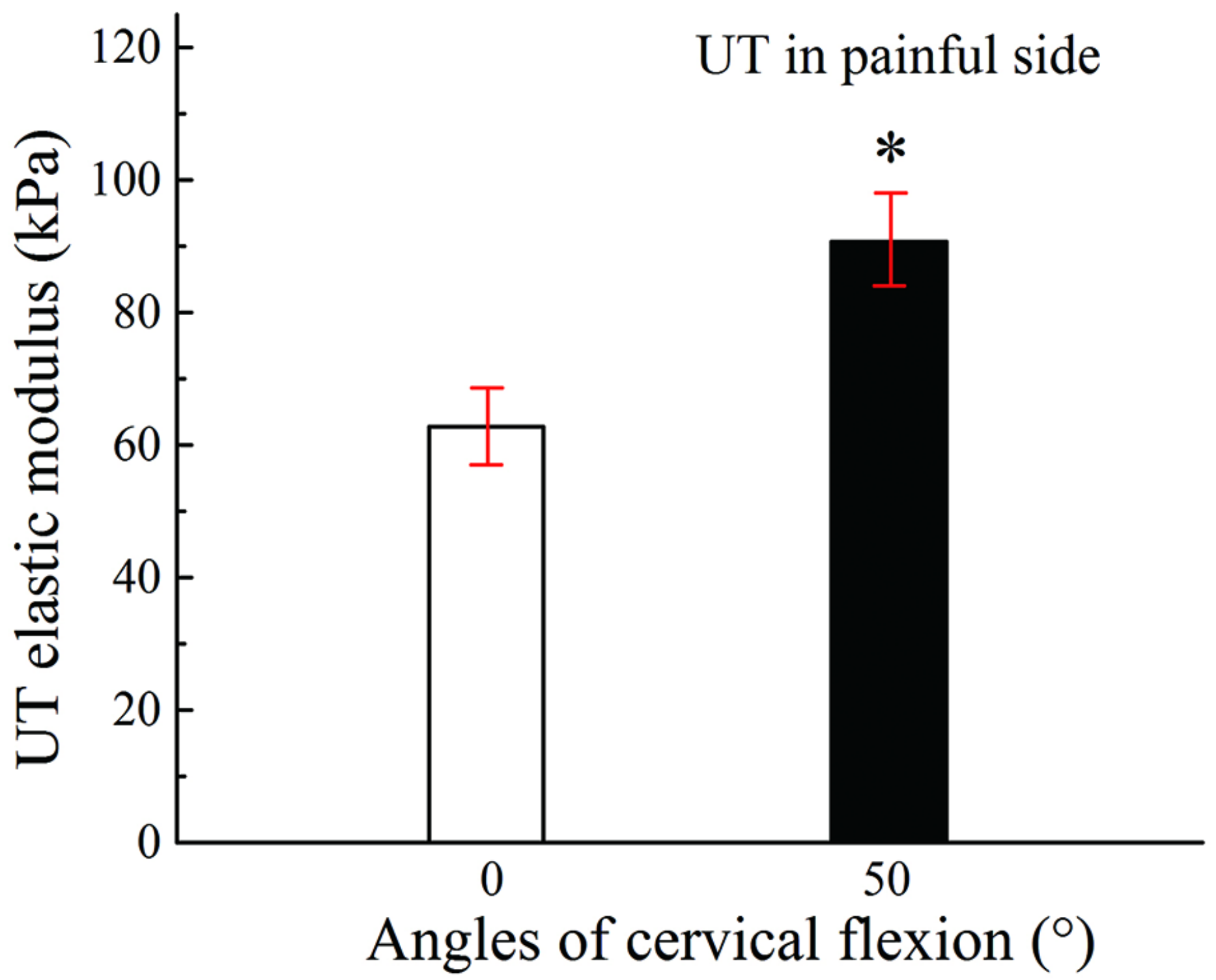

Figure 3

Mean \pm standard deviation for the upper trapezius shear modulus were examined during cervical flexion at $0^{\circ}$ (white bar) and $50^{\circ}$ (black bar). * Denotes significant difference between groups. $(\mathrm{P}<0.05)$. 


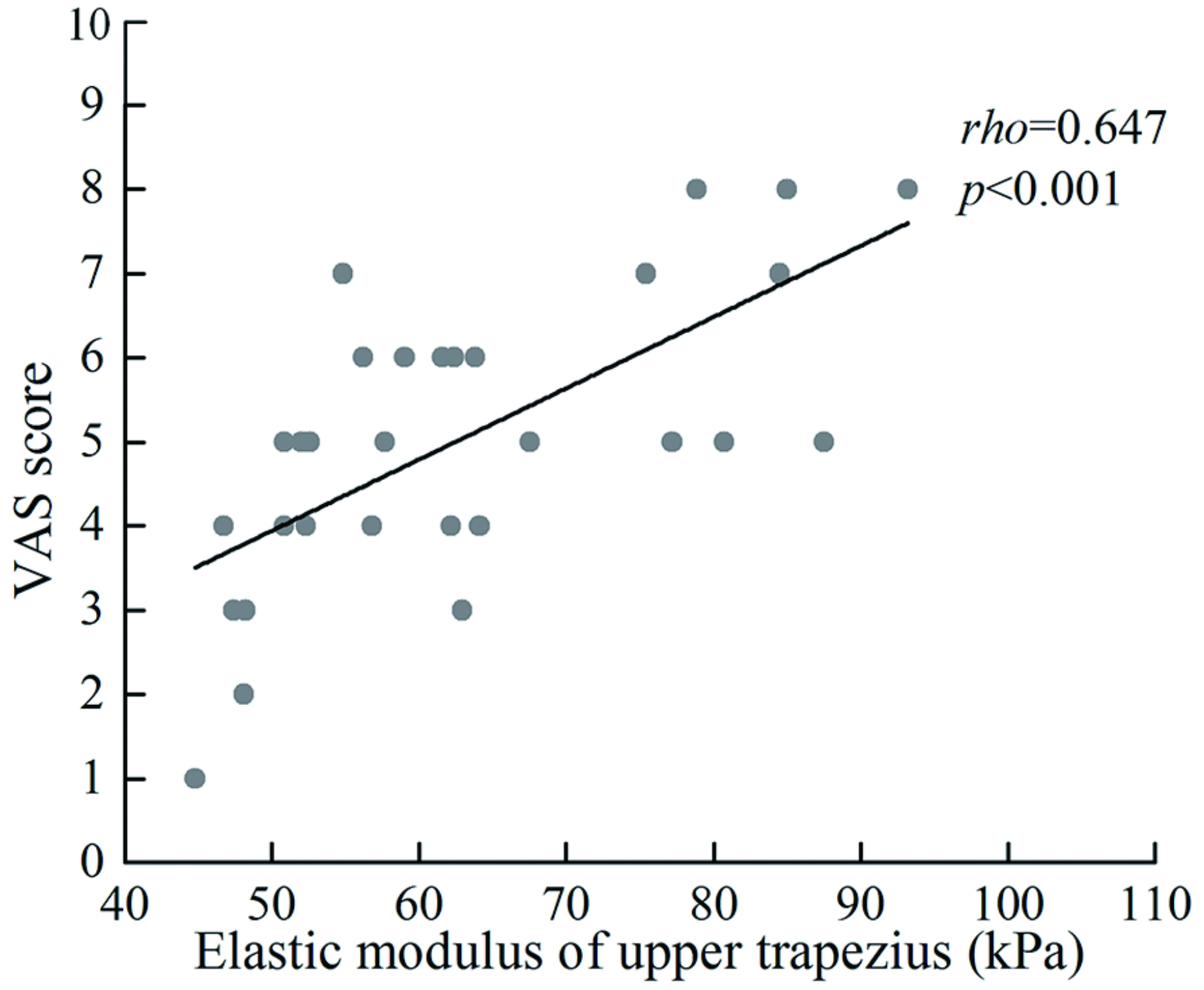

Figure 4

Correlation between intensity of pain and elasticity of the upper trapezius. 